\title{
Aggregator Operation in the Balancing Market Through Network-Constrained Transactive Energy
}

Hu, Junjie; Yang, Guangya; Ziras, Charalampos; Kok, Koen

Published in:

IEEE Transactions on Power Systems

Link to article, DOI:

10.1109/TPWRS.2018.2874255

Publication date:

2019

Document Version

Peer reviewed version

Link back to DTU Orbit

Citation (APA):

Hu, J., Yang, G., Ziras, C., \& Kok, K. (2019). Aggregator Operation in the Balancing Market Through NetworkConstrained Transactive Energy. IEEE Transactions on Power Systems, 34(5), 4071-4080.

https://doi.org/10.1109/TPWRS.2018.2874255

\section{General rights}

Copyright and moral rights for the publications made accessible in the public portal are retained by the authors and/or other copyright owners and it is a condition of accessing publications that users recognise and abide by the legal requirements associated with these rights.

- Users may download and print one copy of any publication from the public portal for the purpose of private study or research.

- You may not further distribute the material or use it for any profit-making activity or commercial gain

- You may freely distribute the URL identifying the publication in the public portal 


\title{
Aggregator Operation in the Balancing Market Through Network-Constrained Transactive Energy
}

\author{
Junjie Hu, Member, IEEE, Guangya Yang, Senior Member, IEEE, Charalampos Ziras, Student Member, IEEE, \\ Koen Kok, Senior Member, IEEE
}

\begin{abstract}
The future renewable-based power system will have an increased need for balancing power. Prosumers, having both generation and consumption capabilities, are expected to provide balancing power to the grid, if their flexibility can be appropriately managed. Meanwhile, undesirable line congestions and voltage violations may arise in the distribution network, when flexible resources respond to external control or price signals on a large scale. Hence, the development of an effective framework to coordinate flexibility at the distribution system level is of utmost importance. Such a framework should allow for an optimal provision of prosumer balancing power services within the boundaries of local network security constraints. In this study, a balancing market participation framework is proposed, adopting the concept of network-constrained transactive energy, to facilitate the interactions between the transmission system operator and aggregators who manage prosumer energy profiles. This framework retains user privacy and complies with the current market setup, where flexible energy is traded on the spot and balancing markets; however, it is ensured that the resulting energy profiles do not cause problems in the distribution network.
\end{abstract}

Index Terms-Aggregator, Balancing market, Prosumers, Transactive energy, Virtual storage

\section{INTRODUCTION}

The future renewable-based power system will have an increased need for balancing power. As conventional generators are being replaced by variable renewable generation units, the need for flexible resources to perform power system balancing is increasing. These resources include batteries, electric vehicles (EVs) and other controllable devices. The large growth in the installed capacity of photovoltaic units, the developments in the grid-to-vehicle $(\mathrm{G} 2 \mathrm{~V})$ and vehicleto-grid (V2G) technologies and the roll-out of other 'smart' responsive devices makes the transition to the prosumer era in the near future possible [1].

To harvest flexibility from these devices and to make these resources reliable for the power system, the aggregator is a pivotal entity which manages the power profile of prosumers and coordinates their interests with those of the system operators. In [2], [3], EVs are optimally aggregated to provide

This work is funded in part by the National Natural Science Foundation of China (Grant No. 51877078) and in part by Danish EUDP project, (Grant no. EUDP17-I: (12551)), PV+STorage - Operation and Economics.

Junjie is with State Key Laboratory of Alternate Electrical Power System with Renewable Energy Sources (North China Electric Power University), Beijing, China. Guangya and Charalampos are with the Center for Electric Power and Energy, Department of Electrical Engineering, Technical University of Denmark, Denmark. Koen is with Netherlands Organization for Applied Research, TNO, The Hague, The Netherlands. Email: \{junjiehu@ncepu.edu.cn, gyy@elektro.dtu.dk, chazi@elektro.dtu.dk, koen.kok@tno.nl\}. balancing services. The authors in [2] formulated a twostage stochastic linear programming problem to determine the optimal bidding strategy of an EV aggregator in the day-ahead (DA) and balancing markets. The method takes the hourly DA energy and regulating clearing price as deterministic parameters and introduces scenarios for two uncertainties: 1) realtime deployed energy to the contracted regulation capacity; 2) real-time energy purchase prices. The optimal participation of an EV aggregator in the DA energy and balancing markets is also studied in [3], where the battery degradation cost is considered. However, both studies [2], [3] do not consider the V2G option, which is expected to gain importance in the near future, considering the latest technology developments [4].

Besides EVs, other forms of aggregation, such as heat pumps [5], or microgrid aggregators [6] are also proposed to participate in the balancing market. In [6], diversified distributed energy resource (DER) units and the associated market mechanism design within the microgrid aggregator concept are investigated. As described in [7]-[9], flexible resources can be categorized into a few groups based on characteristics such as power capacity, energy capacity, the desired energy level at a specific deadline, and minimum run time. A recent study [10] developed a generalized battery model to characterize the flexibility of building loads and energy storage, which is used in an optimal coordination algorithm to provide frequency regulation and spinning reserves.

Although the active participation of DERs ${ }^{1}$ through aggregators is regarded as an important means to retain the power system balance, it has been found that congestion and voltage violations may occur in the distribution network when large amounts of DERs participate in the DA and balancing markets, due to the simultaneous response to external prices [11]. To solve the operational conflicts between the transmission system operator (TSO) and the distribution network operator (DNO) in terms of using DERs flexibility, innovative approaches are needed. Traditionally, the distribution system is kept secure by centralized control actions, e.g. the DNO centrally manages the status of key devices, such as breakers. For a number of reasons, such as market participation conflicts, scalability, complexity and privacy, it is hard for a DNO to directly control large numbers of DERs. Consequently, other mechanisms are required to control DERs and avoid problems in the distribution grid's operation; transactive energy is such an approach. Transactive energy is a form of market-based

\footnotetext{
${ }^{1}$ In the context of this paper, let the term DER encompass all flexible devices connected to the distribution grid, including generation, demand response and storage units.
} 
control technology that has been studied since several years [12], [13] and has been applied to several applications in the smart grid paradigm [14], [15]. Its intent is to achieve an equilibrium via exchanging transaction-based information about generation, consumption, and constraints using a scalable and distributed mechanism that can be standardized in the form of a market protocol.

Building forth on the previously developed networkconstrained transactive energy (NCTE) framework [16], this paper develops an operational framework to enable the participation of aggregators in the balancing market. The model aims to maximize the aggregators' operational profits in the intraday and balancing markets, considering the characteristics and flexibility of prosumers, while maintaining the security and increasing the utilization of the distribution grids. The main contribution of this study is twofold. First, DER flexibility of the prosumers associated with a particular aggregator is modelled as a single virtual storage (VS), representing generation/consumption of each prosumer as charging/discharging the VS; the model considers the possibility of changing power flow direction (between charging and discharging) through the rescheduling of VS compared to the initial schedule and takes it into account in the state of charge (SOC) calculation, which is a significant difference compared to existing works. Second, a receding horizon optimization model of the aggregators' participation in the balancing market is developed, which is compatible with the current market setup and takes into account the latest balancing price predictions as well as other updated information such as the SOC. Aggregators negotiate in a transactive, market-based manner with the DNO to resolve operational problems which may be caused by the aggregators' participation in the balancing market.

The remainder of the paper is organized as follows. In Section II, the system as well as the key actors are introduced. Section III presents the modelling method of the aggregators' optimal participation in the balancing market and the corresponding transactive energy interaction with the DNO. Section IV presents case studies to illustrate the performance of the proposed method. Finally, a discussion and conclusions are presented in Section V.

\section{AgGREgAtor's MARKET PARTICIPATION FRAMEWORK}

Fig. 1 presents the structure of the proposed system, where multiple aggregators interact with prosumers, the DNO, and the distribution independent system operator (DISO) to participate in the balancing market at the transmission level while avoiding grid congestion and voltage violations at the distribution level. Each aggregator represents the interests of a group of prosumers and aims at minimizing their operating costs by optimally participating in the spot and balancing markets. These operations should comply with the DNO's network security constraints, which will be guaranteed by the transactive-energy approach. The DISO is therefore proposed as an independent system operator who coordinates the aggregators and the DNO's operational interests. If there is a violation by the actions of the aggregators in the balancing market, congestion prices will be generated by the DISO to resolve the problem. Otherwise, the power reschedules of the aggregators will be accepted by the DNO and approved by the DISO.

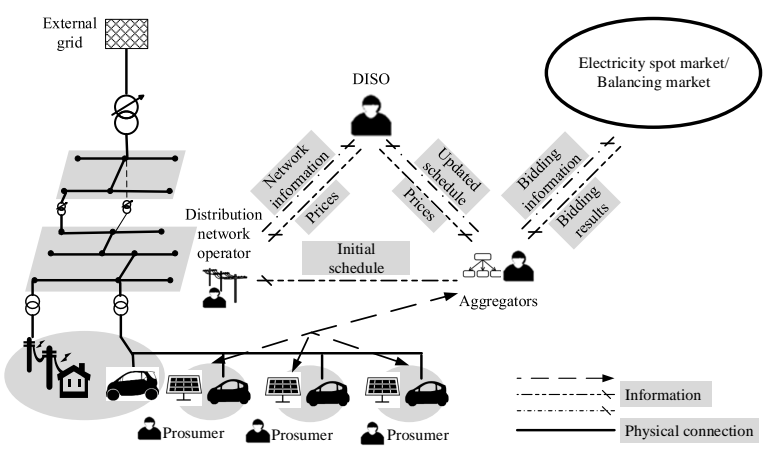

Fig. 1: Transactive energy-enabled operation in a prosumerbased distribution system

Following the European Network of Transmission System Operators (ENTSO-E) definition, balancing refers to the situation after markets have closed (gate closure), in which a TSO acts to ensure that demand is equal to supply, in and close to real time. In Nordic Europe, and in particular the Danish market, the balancing market is divided into two parts: a regulating and a balancing power market. On the former, the Danish TSO buys/sells regulating power from/to the players in the delivery hour on the basis of bids for upward and downward regulation submitted by the players. On the latter, the TSO buys/sells balancing power from/to the players in order to neutralize imbalances incurred by them. Contrary to the procedure applicable to regulating power, the result of these trades is not calculated till after the delivery hour when metered data is available and the imbalances have been quantified. In addition, a player's participation in the regulating power market follows one of two different models: 1) the player can conclude an agreement with Energinet.dk, the Danish TSO on keeping manual reserve available and therefore receives an availability payment in return. When activated, it receives an additional energy payment; 2) the player can refrain from concluding such an agreement (non-agreement approach), instead placing bids as the player sees fit. Upon activation, the player receives only an energy payment.

In the regulating power market operation, the players' bids are submitted to the TSO, who collects all bids to form a pool. The bids may cover an entire day of operation, but the player can adjust them up to 45 minutes prior to the upcoming delivery hour. The upward and downward regulation bids must be separate and consist of price (DKK/MWh) and volume (MW). When needed, the bids with the lowest prices will normally be the first ones to be activated (unless bids are bypassed in the merit-order due to congestion in the transmission system). Orders for upward or downward regulation are communicated either on 5-min intervals or by direct activation without any exchange of schedules.

In this study the following assumptions are made for the market operation: 1) the market scenario we considered for the participation of aggregators is similar to the regulating 


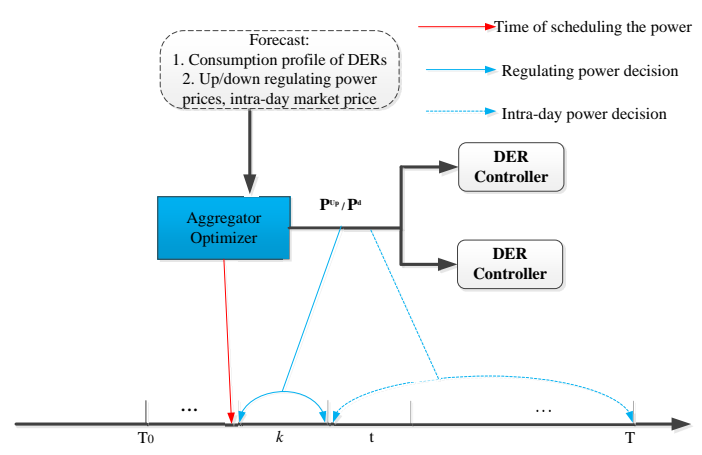

Fig. 2: Illustration of the aggregator's participation in the balancing and intra-day market.

power market; 2) the aggregators use the non-agreement approach to participate in the balancing market; 3) aggregators have perfect predictions on the incoming one-hour upward and downward regulation price and then decide the optimal power profile, which will be interpreted as bids which will be sent to the TSO; 4) the upward and downward regulation power bids will be fully activated by the TSO in one hour; 5 ) to re-balance the energy requirements of virtual storage, intra-day market participation is included in the aggregator's optimization problem.

\section{System ModelLing}

In this section, we first present the modelling of the optimization problem of the aggregators, who aim at maximizing their profits in the balancing market. Second, the aggregators' operation within the NCTE is described and then the DNO's optimization problem is formulated. Next, the centralized variant of the overall problem is presented, as a social cost minimization problem which compromises the interests of the aggregators and the DNO. Finally, we present the NCTE approach for solving the problem in a transactive manner.

\section{A. Aggregator's Operation in the Balancing Market}

We model the prosumers' flexibility as virtual storage (VS), in which the energy and power constraints are considered. Furthermore, we model the aggregated power and equivalent SOC of each aggregator's virtual storage connected at each bus $i$ of the grid. In this subsection we present the aggregator's optimization problem, considering a fixed DA schedule; $P_{i, t}^{\mathrm{ch}}$ denotes the charging power of the aggregated virtual storage sources at bus $i$ and time step $t$ and is non-negative, whereas $P_{i, t}^{\text {dis }}$ denotes the discharging power and is non-positive. This DA schedule corresponds to the initial DA schedule of the aggregator or is the result from re-scheduling via the intraday market. This schedule will serve as a baseline demand, upon which up and down regulating power is offered to the market. For notation simplicity, at this subsection, we omit the index for each aggregator, which is later denoted by $j$.

We denote by $P_{i, k}^{\mathrm{up}}$ the offered up-regulating power at time step $k$, which corresponds to load reduction and is a nonnegative variable. To further simplify notation, we use the

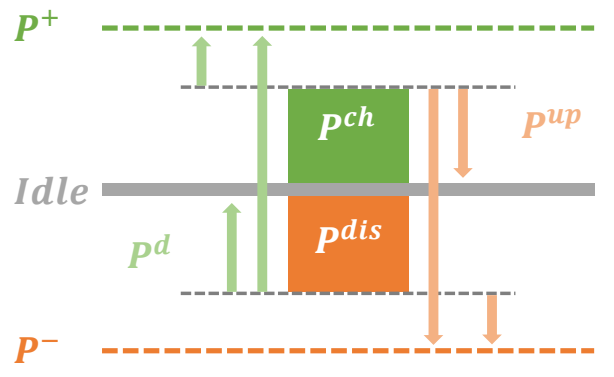

Fig. 3: Illustration of VS's power direction when providing up and down regulating power. Both $P^{\text {up }}$ and $P^{\mathrm{d}}$ are defined as non-negative variables.

same variable name for load reductions for $t>k$ in the intra-day market. Consequently, values of $P_{i, t}^{\text {up }}$ for $t=k$ refer to the offered regulating power and $t>k$ to the intra-day reschedules. The same reasoning applies to $P_{i, k}^{\mathrm{d}}$ and downregulation. The time indexes and a schematic representation of an aggregator's participation in the balancing and intra-day markets is shown in Fig. 2. We must note that the two sets of variables are mutually exclusive, i.e. if one takes a non-zero value the other must be zero.

As shown in Fig. 3, where $P^{-}$and $P^{+}$denote the maximum discharging and charging power capacity respectively, the charging/discharging mode can change after providing up or down regulating power. This change in charging/discharging mode has to be taken into account in the SOC calculation, which results in the introduction of integer variables to determine the VS's mode. We introduce a set of variables $\delta$ to represent the necessary logical constraints, which are summarized in Table I.

TABLE I: Binary variables explanation

\begin{tabular}{c|c}
\hline Variable & Description \\
\hline$\delta^{1}$ & Up-regulating power is offered and $P^{\mathrm{up}} \geq 0, P^{\mathrm{d}}=0$ \\
$\delta^{2}$ & Down regulating power is offered and $P^{\mathrm{d}} \geq 0, P^{\mathrm{up}}=0$ \\
$\delta^{3}$ & VS is in charging mode and $P^{\mathrm{ch}}-P^{\mathrm{up}} \geq 0$ \\
$\delta^{4}$ & VS is in discharging mode and $P^{\mathrm{ch}}-P^{\mathrm{up}}<0$ \\
$\delta^{5}$ & VS is in charging mode and $P^{\mathrm{dis}}+P^{\mathrm{d}} \geq 0$ \\
$\delta^{6}$ & VS is in discharging mode and $P^{\mathrm{dis}}+P^{\mathrm{d}}<0$ \\
\hline
\end{tabular}

If $\lambda_{k}^{\mathrm{up}}$ and $\lambda_{k}^{\mathrm{d}}$ are the up and down regulation prices respectively at time step $k$, and $\lambda_{t}^{\mathrm{DA}}$ are the DA prices (for simplicity we assume the intra-day prices are equal to the DA prices) for the time steps $t$, the optimization problem for aggregator $j$ then takes the following form 


$$
\begin{array}{ll}
\min _{\delta, \mathbf{P}^{\mathrm{up}}, \mathbf{P}^{\mathrm{d}}} & \sum_{i \in \Omega_{j}}\left[\left(-P_{i, k}^{\mathrm{up}} \lambda_{k}^{\mathrm{up}}+P_{i, k}^{\mathrm{d}} \lambda_{k}^{\mathrm{d}}\right)+\right. \\
& \left.\sum_{t=k+1}^{T}\left(-P_{i, t}^{\mathrm{up}}+P_{i, t}^{\mathrm{d}}\right) \lambda_{t}^{\mathrm{DA}}\right] \\
& \delta_{i, t}^{1}, \delta_{i, t}^{2}, \delta_{i, t}^{3}, \delta_{i, t}^{4}, \delta_{i, t}^{5}, \delta_{i, t}^{6} \in\{0,1\} \\
& \delta_{i, t}^{1}+\delta_{i, t}^{2} \leq 1 \\
& 0 \leq P_{i, t}^{\mathrm{up}} \leq \delta_{i, t}^{1}\left(P_{i}^{-}+P_{i, t}^{\mathrm{ch}}+P_{i, t}^{\mathrm{dis}}\right) \\
& 0 \leq P_{i, t}^{\mathrm{d}} \leq \delta_{i, t}^{2}\left(P_{i}^{+}-P_{i, t}^{\mathrm{ch}}-P_{i, t}^{\mathrm{dis}}\right) \\
& \delta_{i, t}^{3}+\delta_{i, t}^{4}=1, \delta_{i, t}^{5}+\delta_{i, t}^{6}=1 \\
& \delta_{i, t}^{3}\left(P_{i, t}^{\mathrm{ch}}-P_{i, t}^{\mathrm{up}}\right) \geq 0, \delta_{i, t}^{4}\left(P_{i, t}^{\mathrm{ch}}-P_{i, t}^{\mathrm{up}}\right) \leq 0 \\
& \delta_{i, t}^{5}\left(P_{i, t}^{\mathrm{dis}}+P_{i, t}^{\mathrm{d}}\right) \geq 0, \delta_{i, t}^{6}\left(P_{i, t}^{\mathrm{dis}}+P_{i, t}^{\mathrm{d}}\right) \leq 0 \\
& S O C_{i, t+1}=S O C_{i, t}+\eta_{+}\left(1 \mathrm{c} P_{i, t}^{\mathrm{ch}}-P_{i, t}^{\mathrm{up}}\right) \delta_{i, t}^{3} \\
& +\left(1 / \eta_{-}\right)\left(P_{i, t}^{\mathrm{ch}}-P_{i, t}^{\mathrm{up}}\right) \delta_{i, t}^{4}+\eta_{+}\left(P_{i, t}^{\mathrm{dis}}+P_{i, t}^{\mathrm{d}}\right) \delta_{i, t}^{5} \\
& +\left(1 / \eta_{-}\right)\left(P_{i, t}^{\mathrm{dis}}+P_{i, t}^{\mathrm{d}}\right) \delta_{i, t}^{6} \\
& S O C_{i, t}^{\text {min }} \leq S O C_{i, t} \leq S O C_{i, t}^{\text {max }} \\
& S O C_{i, T}=S O C_{i}^{\mathrm{des}}
\end{array}
$$

where $\Omega_{j}$ is the set mapping the aggregated VSs of aggregator $j$ at bus $i$ to the grid. $\eta_{+}$and $\eta_{-}$represent the charging and discharging efficiency respectively. Finally, $S O C^{\text {des }}$ is the desired equivalent SOC value at the end of the optimization horizon and $T$ is the number of the horizon's time steps. We use bold fonts to denote a vector of variables such as $\delta$, in contrast to scalar variables such as $\delta_{i, t}^{1}$.

The formulation in (1) contains bi-linear terms, such as $\delta_{i, t}^{3} \cdot P_{i, t}^{\text {up }}$ in the optimization problem. Here we linearize the problem by employing the "big $\mathrm{M}$ " technique [17]. To linearize the above formulated problem we introduce variable $z_{i, t}^{1}=\delta_{i, t}^{3} P_{i, t}^{\mathrm{up}} . P_{i, t}^{\mathrm{up}}$ is lower bounded by 0 and upper bounded by $\left(P_{i}^{-}+P_{i, t}^{\text {ch }}+P_{i, t}^{\mathrm{dis}}\right)$. Therefore, the following constraints must be also satisfied

$$
\begin{array}{r}
z_{i, t}^{1} \leq\left(P_{i}^{-}+P_{i, t}^{\mathrm{ch}}+P_{i, t}^{\mathrm{dis}}\right) \delta_{i, t}^{3} \\
0 \leq z_{i, t}^{1} \leq P_{i, t}^{\text {up }} \\
z_{i, t}^{1} \geq P_{i, t}^{\text {up }}-\left(1-\delta_{i, t}^{3}\right)\left(P_{i}^{-}+P_{i, t}^{\text {ch }}+P_{i, t}^{\text {dis }}\right)
\end{array}
$$

Similarly, we introduce variables $z_{i, t}^{2}=\delta_{i, t}^{4} P_{i, t}^{\text {up }}, z_{i, t}^{3}=$ $\delta_{i, t}^{5} P_{i, t}^{\mathrm{d}}$ and $z_{i, t}^{4}=\delta_{i, t}^{6} P_{i, t}^{\mathrm{d}}$, with their corresponding constraints. Finally, the reformulated optimization problem takes the following form

$$
\begin{aligned}
& \min _{\boldsymbol{\delta}, \boldsymbol{z}, \mathbf{P}^{\mathrm{up}}, \mathbf{P}^{\mathrm{d}}} \sum_{i \in \Omega_{j}}\left[\left(-P_{i, k}^{\mathrm{up}} \lambda_{i, k}^{\mathrm{up}}+P_{i, k}^{\mathrm{d}} \lambda_{i, k}^{\mathrm{d}}\right)+\right. \\
& \left.\sum_{t=k+1}^{T}\left(-P_{i, t}^{\mathrm{up}}+P_{i, t}^{\mathrm{d}}\right) \lambda_{i, t}^{\mathrm{DA}}\right] \\
& \text { s.t. } \quad(1 \mathrm{~b})-(1 \mathrm{f}),(1 \mathrm{j}),(1 \mathrm{k}) \\
& z_{i, t}^{1}, z_{i, t}^{2}, z_{i, t}^{3}, z_{i, t}^{4} \geq 0 \\
& \delta_{i, t}^{3} P_{i, t}^{\mathrm{ch}}-z_{i, t}^{1} \geq 0, \quad \delta_{i, t}^{4} P_{i, t}^{\mathrm{ch}}-z_{i, t}^{2} \leq 0 \\
& \delta_{i, t}^{5} P_{i, t}^{\mathrm{dis}}+z_{i, t}^{3} \geq 0, \quad \delta_{i, t}^{6} P_{i, t}^{\mathrm{dis}}+z_{i, t}^{4} \leq 0 \\
& z_{i, t}^{1} \leq\left(P_{i}^{-}+P_{i, t}^{\mathrm{ch}}+P_{i, t}^{\mathrm{dis}}\right) \delta_{i, t}^{3} \\
& z_{i, t}^{2} \leq\left(P_{i}^{-}+P_{i, t}^{\mathrm{ch}}+P_{i, t}^{\mathrm{dis}}\right) \delta_{i, t}^{4} \\
& z_{i, t}^{3} \leq\left(P_{i}^{+}-P_{i, t}^{\mathrm{ch}}-P_{i, t}^{\mathrm{dis}}\right) \delta_{i, t}^{5} \\
& z_{i, t}^{4} \leq\left(P_{i}^{+}-P_{i, t}^{\mathrm{ch}}-P_{i, t}^{\mathrm{dis}}\right) \delta_{i, t}^{6} \\
& z_{i, t}^{1} \leq P_{i, t}^{\mathrm{up}}, \quad z_{i, t}^{2} \leq P_{i, t}^{\mathrm{up}} \\
& z_{i, t}^{3} \leq P_{i, t}^{\mathrm{d}}, \quad z_{i, t}^{4} \leq P_{i, t}^{\mathrm{d}} \\
& z_{i, t}^{1} \geq P_{i, t}^{\mathrm{up}}-\left(1-\delta_{i, t}^{3}\right)\left(P_{i}^{-}+P_{i, t}^{\mathrm{ch}}+P_{i, t}^{\mathrm{dis}}\right) \\
& z_{i, t}^{2} \geq P_{i, t}^{\text {up }}-\left(1-\delta_{i, t}^{4}\right)\left(P_{i}^{-}+P_{i, t}^{\text {ch }}+P_{i, t}^{\text {dis }}\right) \\
& z_{i, t}^{3} \geq P_{i, t}^{\mathrm{d}}-\left(1-\delta_{i, t}^{5}\right)\left(P_{i}^{+}-P_{i, t}^{\mathrm{ch}}-P_{i, t}^{\mathrm{dis}}\right) \\
& z_{i, t}^{4} \geq P_{i, t}^{\mathrm{d}}-\left(1-\delta_{i, t}^{6}\right)\left(P_{i}^{+}-P_{i, t}^{\mathrm{ch}}-P_{i, t}^{\mathrm{dis}}\right) \\
& S O C_{i, t+1}=S O C_{i, t}+\eta_{+}\left(P_{i, t}^{\mathrm{ch}} \delta_{i, t}^{3}-z_{i, t}^{1}\right) \\
& +\left(1 / \eta_{-}\right)\left(P_{i, t}^{\mathrm{ch}} \delta_{i, t}^{4}-z_{i, t}^{2}\right)+\eta_{+}\left(P_{i, t}^{\mathrm{dis}} \delta_{i, t}^{5}+z_{i, t}^{3}\right) \\
& +\left(1 / \eta_{-}\right)\left(P_{i, t}^{\mathrm{dis}} \delta_{i, t}^{6}+z_{i, t}^{4}\right)
\end{aligned}
$$

The optimization produces a schedule at each bus $i$ for each time step $t$. We denote this aggregated and unconstrained schedule by $P_{i, t}^{\text {unc }}$, since so far DNO constraints are not considered. In this sense, $P_{i, t}^{\text {unc }}$ reflects the optimal reschedules of the aggregators in the remaining optimization horizon, which must be modified (via the transactive energy mechanism with the DNO and the DISO), if the optimal schedules of the aggregators violate the DNO's operational constraints. $P_{i, t}^{\text {unc }}$ is defined as

$$
P_{i, t}^{\mathrm{unc}}=P_{i, t}^{\mathrm{dis}}+P_{i, t}^{\mathrm{ch}}+P_{i, t}^{\mathrm{d}, *}-P_{i, t}^{\mathrm{up}, *},
$$

where $*$ indicates that these values correspond to the solution of the aggregator's unconstrained (from DNO constraints) optimization problem. Solving (5) may result in very low scheduled power values, which may not be feasible by the units. However, in contrast to generators which have strict minimum loading requirements, this problem is not so prominent in the case of inverter-equipped units such as batteries or EVs. Additionally, due to the aggregated nature of the considered VS, it is possible to track a low aggregated charging/discharging schedule by distributing the setpoints to the devices accordingly. The low-level, real-time control is outside the scope of this paper, but we believe that it is possible to design control policies to overcome such issues.

\section{B. Aggregator's Operation in NCTE}

In NCTE, the aggregator's goal is to minimize the deviations from its calculated optimal schedule. Let $P_{i, t}^{\text {ch,c }}$ and $P_{i, t}^{\text {dis,c }}$ 
denote the new, mutually exclusive, 'constrained' values for the aggregated charging and discharging respectively. It is common to introduce binary variables to impose the mutual exclusiveness of the charging/discharging mode. However, as indicated in [10], [18] the binary variables are unnecessary if $\eta_{+} \eta_{-}<1$, which is the case in real applications, and thus we do not need to include binary variables in the formulation. We propose a quadratic cost function, denoted by $\mu_{j}$ for aggregator $j$, which represents the deviations cost caused by the DNO's constraints

$$
\begin{array}{ll}
\min _{\delta, \mathbf{p}^{\mathrm{ch}, \mathrm{c}}, \mathbf{p}^{\mathrm{dis}, \mathrm{c}}} & \mu_{j}=\sum_{t=k}^{T} \sum_{i \in \Omega_{j}} C_{i, t}\left(P_{i, t}^{\mathrm{ch,c}}+P_{i, t}^{\mathrm{dis}, \mathrm{c}}-P_{i, t}^{\mathrm{unc}}\right)^{2} \\
\text { s.t. } & S O C_{i, t+1}=S O C_{i, t}+\eta_{+} P_{i, t}^{\mathrm{ch}, \mathrm{c}}+\left(1 / \eta_{-}\right) P_{i, t}^{\mathrm{dis}, \mathrm{c}}
\end{array}
$$

$$
\begin{aligned}
& 0 \leq P_{i, t}^{\text {ch,c }} \leq P_{i}^{+} \\
& 0 \geq P_{i, t}^{\text {dis, }} \geq-P_{i}^{-}
\end{aligned}
$$

Note that $\mathbf{P}^{\mathrm{ch}, \mathrm{c}}$ and $\mathbf{P}^{\mathrm{dis}, \mathrm{c}}$ represent the new, constrained schedules in a similar way $\mathbf{P}^{\mathrm{ch}}$ and $\mathbf{P}^{\mathrm{dis}}$ were defined. $C_{i, t}$ are weighting factors which are associated with the power differences; the larger the value of $C_{i, t}$, the smaller the preferred deviation from the unconstrained value. Furthermore, we need to point out that it should be very careful when removing the binary variables from the charging and discharging model. In [18], the authors did not provide a rigorous argument, while the study [10] did give an argument, it only presented a sufficient condition of removing the binary variables if the objective function of an optimization problem has monotonically increasing characteristic. The function used in our study does not have this feature, however, according to our extensive simulation, it is learned that the optimal value of charging and discharging decision variable will not appear simultaneously in a relaxed condition, e.g., by setting the absolute value of $\left(P_{c h} * P_{d i s}\right)$ to be bigger than 0.01 .

\section{DNO's Operational Objective and Constraints}

The DNO's objective is to supply the power needed by the aggregators and facilitate their participation in the regulating market, as well as respect grid constraints which include line thermal constraints and bus voltage constraints. For this reason we use a quadratic cost function which penalizes the deviations of the capacity allocated by the DNO from the optimal unconstrained schedules of the aggregators. We use a simplified linearization method of the power flows to account for the effect of the aggregators' modified schedules on voltage levels [16], [19]. To do so, first the Jacobian matrix of the network is calculated, as well as the base voltage levels $U_{i, t}^{0}$, by considering only the conventional load of the system. The rescheduled profiles of the aggregators must satisfy the following constraints:

$$
\begin{array}{ll}
\min _{\mathbf{p}^{\mathrm{DNO}}} & h=\sum_{t=k}^{T} \sum_{i=1}^{N_{B}}\left(P_{i, t}^{\mathrm{DNO}}-\sum_{j=1}^{M} P_{j, i, t}^{\mathrm{unc}}\right)^{2} \\
\text { s.t. } & -P_{\text {trans }, t}^{\max } \leq \sum_{i=1}^{N_{B}} P_{i, t}^{\mathrm{DNO}} \leq P_{\text {trans }, t}^{\mathrm{max}} \\
& U_{i, t}^{\min } \leq U_{i, t}^{0}+J_{\mathbf{2 1}}^{-1} \cdot P_{i, t}^{\mathrm{DNO}} \leq U_{i, t}^{\max }
\end{array}
$$

where $U_{i, t}^{\min }, U_{i, t}^{\max }$ are the minimum and maximum voltage levels respectively, $P_{\text {trans }, t}^{\max }$ is the allocated capacity at the transformer level, $N_{\mathrm{B}}$ is the number of buses and $M$ the numbe of aggregators. Note that in (8c) a submatrix of the inverse Jacobian is used, because we assume that only active power is rescheduled and thus the reactive power increments are zero. Other linearization methods such as [20] can also be used but we found via extensive simulations that the used approximation yields very good results.

\section{Centralized Problem}

From a social welfare maximization point of view, it is desirable to minimize the aggregators cost and the DNO's cost, as well as mitigate any undesirable impact of the aggregators actions on the distribution level. The social welfare maximization problem is mathematically formulated as a social cost minimization problem as follows

$$
\begin{array}{ll}
\min _{\mathbf{P}^{\mathrm{DNO}}, \mathbf{P}^{\mathrm{ch},}, \mathbf{P}^{\mathrm{dis}, \mathrm{c}}} & \sum_{j=1}^{M} \mu_{j}\left(\mathbf{P}_{j}^{\mathrm{ch}, \mathrm{c}}, \mathbf{P}_{j}^{\mathrm{dis}, \mathrm{c}}\right)+h\left(\mathbf{P}^{\mathrm{DNO}}\right), \\
\text { s.t. } & (7 \mathrm{~b})-(7 \mathrm{~d}),(8 \mathrm{~b})-(8 \mathrm{c}) \\
& \sum_{j=1}^{M}\left(P_{j, i, t}^{\mathrm{ch}, \mathrm{c}}+P_{j, i, t}^{\mathrm{dis}, \mathrm{c}}\right)=P_{i, t}^{\mathrm{DNO}}, \forall i, t,
\end{array}
$$

where (9b) is a global constraint for the DNO and all aggregators.

\section{E. Transactive Energy Modelling and Implementation}

1) Transactive energy modelling: We employ a transactive energy modelling approach, where the aforementioned centralized optimization problem is mathematically modelled as its dual problem [21]. Let $\boldsymbol{\lambda}$ denote the set of Lagrange multipliers (LMs) corresponding to (9b). By keeping the rest of the constraints implicit, the Lagrangian function for the centralized problem is

$$
\begin{aligned}
& L\left(\boldsymbol{\lambda}, \mathbf{P}^{\mathrm{ch}, \mathrm{c}}, \mathbf{P}^{\mathrm{dis}, \mathrm{c}}, \mathbf{P}^{\mathrm{DNO}}\right)=\sum_{j=1}^{M} \mu_{j}\left(\mathbf{P}_{j}^{\mathrm{ch}, \mathrm{c}}, \mathbf{P}_{j}^{\mathrm{dis}, \mathrm{c}}\right)+h\left(\mathbf{P}^{\mathrm{DNO}}\right) \\
& +\sum_{t=k}^{T} \sum_{i=1}^{N_{\mathrm{B}}} \lambda(i, t)\left(\sum_{j=1}^{M}\left(P_{j, i, t}^{\mathrm{ch}, \mathrm{c}}+P_{j, i, t}^{\mathrm{dis}, \mathrm{c}}\right)-P_{i, t}^{\mathrm{DNO}}\right)
\end{aligned}
$$

The dual problem is formulated as follows:

$$
\max \left\{\inf _{\mathbf{P}^{\mathrm{ch},}, \mathbf{P}, \mathbf{P}_{\mathrm{dis}, \mathrm{c}}, \mathbf{P}^{\mathrm{DNO}}} L\left(\boldsymbol{\lambda}, \mathbf{P}^{\mathrm{ch}, \mathrm{c}}, \mathbf{P}^{\mathrm{dis}, \mathrm{c}}, \mathbf{P}^{\mathrm{DNO}}\right)\right\}
$$


2) Transactive energy implementation: To solve the dual optimization problem (11), a decomposition method is applied that can decompose the problem into sub-problems. This not only reduces the problem's complexity but also provides a way of distributing the right of decision-making to each actor in the network. The problems include the aggregators' and DNO's optimization problems, coordinated by the DISO by updating the Lagrange multipliers $\lambda(i, t)$. In order to solve the dual optimization problem (11), we apply the sub-gradient method/distributed algorithm [22], [23], which requires multiple iterations of information exchange. In each iteration all actors receive the updated LMs and send their optimal power values to the DISO. Each aggregator's minimization problem can be written as

$$
\min _{\mathbf{P}^{\mathrm{ch}, \mathrm{c}, \mathbf{P}}, \mathbf{P}_{\mathrm{dis}, \mathrm{c}}} \mu_{j}\left(\mathbf{P}^{\mathrm{ch}, \mathrm{c}}, \mathbf{P}^{\mathrm{dis}, \mathrm{c}}\right)+\sum_{t=k}^{T} \sum_{i \in \Omega_{j}} \lambda(i, t)\left(P_{j, i, t}^{\mathrm{ch}, \mathrm{c}}+P_{j, i, t}^{\mathrm{dis}, \mathrm{c}}\right),
$$

s.t. the same aggregator constraints presented in subsection III-B. The DNO's minimization problem is now

$$
\min _{\mathbf{P}^{\mathrm{DNO}}} h\left(\mathbf{P}^{\mathrm{DNO}}\right)-\sum_{t=k}^{T} \sum_{i=1}^{N_{\mathrm{B}}} \lambda(i, t) P_{i, t}^{\mathrm{DNO}},
$$

s.t. the DNO constraints of DNO of subsection III-C. The LMs are updated according to

$$
\begin{aligned}
\lambda_{\omega+1}(i, t)= & \lambda_{\omega}(i, t)+ \\
& \alpha_{\omega} \cdot\left[\sum_{j=1}^{M}\left(P_{j, i, t}^{\mathrm{ch}, \mathrm{c}, *}+P_{j, i, t}^{\mathrm{dis}, \mathrm{c}, *}\right)-P_{i, t}^{\mathrm{DNO}, *}\right],
\end{aligned}
$$

where $\omega$ is the index for the iterations, $P_{j, i, t}^{\text {ch,c,* }}+P_{j, i, t}^{\text {dis,c,* }}$ correspond to the solution of problem (12) and $P_{i, t}^{\mathrm{DNO}, *}$ to the solution of (13). $\alpha_{\omega}$ is a step size, which has to be positive to guarantee convergence. To solve problems (5), (8), (12), (13) we use the Gurobi solver [24] with the YALMIP interface under Matlab [25]. Note that problem (5) is solved once to obtain the unconstrained values. To derive the Jacobian matrix we used MATPOWER [26], a MATLAB power system simulation package. In Fig. 4 a flowchart which illustrates the different steps of the proposed framework and the connection of the different optimization problems is shown.

In practice, the information exchanges can be facilitated by a system shown in Fig. 1, which shows the implementation of the proposed network-constrained transactive control. In such a framework, the DISO manages the congestion prices (i.e. the LMs) and sends updated values to the DNO and the aggregators to achieve convergence. The DISO could be operated as a third party. Such a party would be economically feasible if a substantial number of DERs are connected on the distribution networks level and if this independent party was used to provide such services to different DNOs.

\section{CASE STUdY}

In this section we apply the NCTE method to solve congestion and voltage violation problems caused by the participation of aggregators in the balancing market, using a real Danish low voltage distribution network. In subsection IV-A, all the

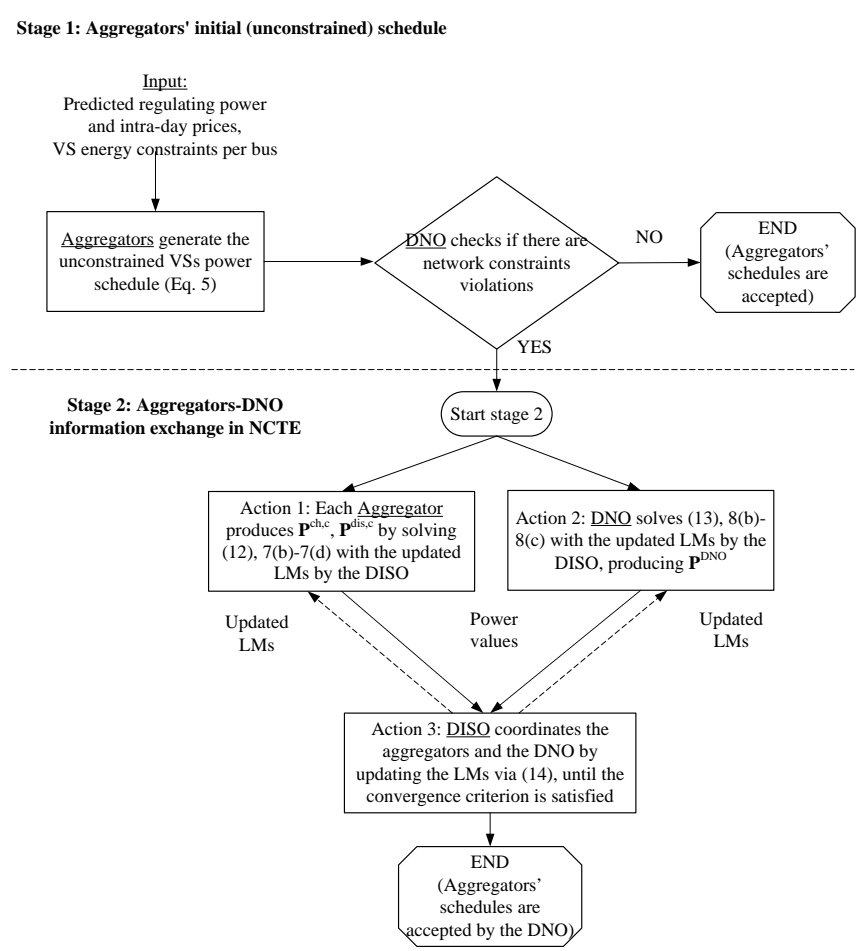

Fig. 4: NCTE implementation framework.

relevant parameters of the virtual storage and the DA, as well as the up/down regulating power prices are described. Moreover, the distribution network used in this study is presented. Then, in subsection IV-B, the unconstrained schedules, i.e., the aggregators' optimal schedules for offering balancing power without any DNO grid constraints is presented. Thirdly, in subsection IV-C, the results of applying NCTE to resolve grid constraints violations are shown by comparing the aggregated power before and after NCTE. Finally, in subsection IV-D, we apply NCTE in a receding horizon optimization problem, to show how aggregators can continuously participate in the balancing market by updating their power profiles at every time step the transactive mechanism is applied.

\section{A. Case Specification}

1) Virtual storage model parameters: The VS technical specifications take the parameter values from EV batteries, since EV batteries contain the full features of a virtual storage model considering V2G and G2V. The model can adopt another set of parameters to reflect the operation of an actual prosumer. It is assumed that all the EVs are affiliated to either aggregator 1 (Agg.1) or aggregator 2 (Agg.2). Agg.1 and Agg.2 are both operating 9 EVs each. The weighting factors $C$ in (7) are set equal 1 for both aggregators. The other parameters of EV charging are the following:

- Battery capacity $E^{\text {cap }}$ is set equal to $30 \mathrm{kWh}$.

- The initial $S O C$ is set to $50 \%$ of the battery capacity.

- $S O C^{\max }$ is set to $90 \%$ of the battery capacity.

- $S O C^{\text {min }}$ is set to $10 \%$ of the battery capacity.

- $S O C^{\text {des }}$ is set to $90 \%$ of the battery capacity.

- $P^{+}=P^{-}=10 \mathrm{~kW}, \eta_{+}=0.9$ and $\eta_{-}=0.9$. 


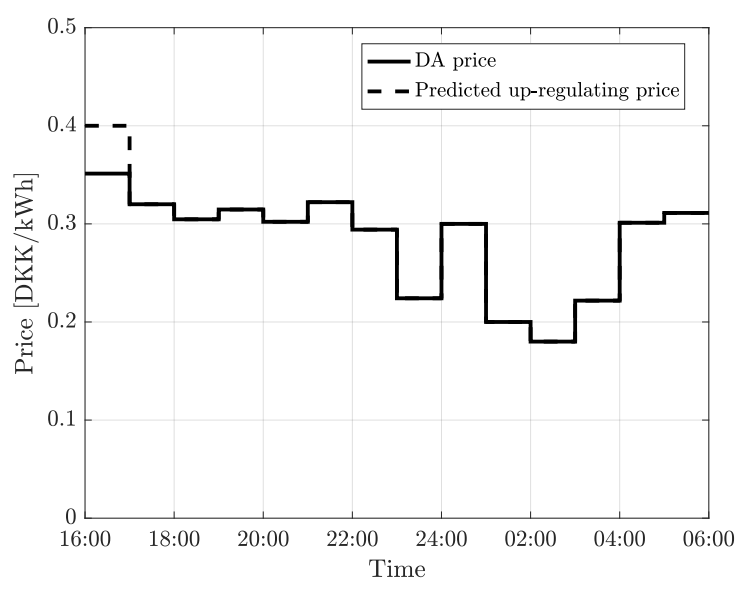

Fig. 5: Electricity energy price, an example from NordPool

2) DA and predicted up/down-regulating power price: We assume that all EVs are parked and available from 16.00 to 06.00 and we use an hourly time interval. The hourly DA market prices from 16.00 to 06.00 are assumed to be known to the aggregators; the used prices ${ }^{2}$ are shown in Fig. 5 and are used for generating the EVs DA charging schedule. In addition, the DA prices will also be used as intraday market prices for the aggregators' rescheduling actions. Besides the DA electricity prices, an example of predicted up/down regulating power prices is also presented in Fig. 5. Note that at each time slot of the optimization's receding horizon, updated predictions of up-/down-regulating prices would be used in practice.

3) Distribution network and control parameters: A representative Danish distribution grid is illustrated in Fig. 6, where 72 households are connected to the feeders: 51 households are attached to the left branch and 21 households to the right branch of the network. The base load of the uncontrollable load is assumed to be known by the DNO. With the base load known, the DNO can calculate the base voltage per bus $U_{i, t}^{0}$. In all time slots, the power transformer capacity allocated to the aggregators is $150 \mathrm{~kW}$ and the minimum/maximum voltage $U_{\min } / U_{\max }$ per bus is assumed to be 0.90 and $1.1 \mathrm{pu}$ respectively. The initial LMs are all set equal to zero and the updated values are sent per iteration to the aggregators and the DNO; $\alpha_{\omega}=0.4$ is chosen for the LMs update.

\section{B. Aggregator's Optimal Schedule for the Balancing Market - Unconstrained Schedules}

At first we calculate the DA charging/discharging profile for the aggregators at each bus based on the commonly used quadratic-based optimization of [16], which we omit due to space limitations. As shown in Fig. 7, the total DA power is distributed along the whole horizon but is mainly located in early morning time slots since the DA price is relatively low. For simplicity, we refer to the sum of the DA schedule and the regulating power (or the intra-day reschedules) as the updated power schedules. Due to the price incentive and the predicted

\footnotetext{
${ }^{2}$ The electricity price assumed here is drawn from the real electricity price from NordPool spot market (http://www.nordpoolspot.com/)
}

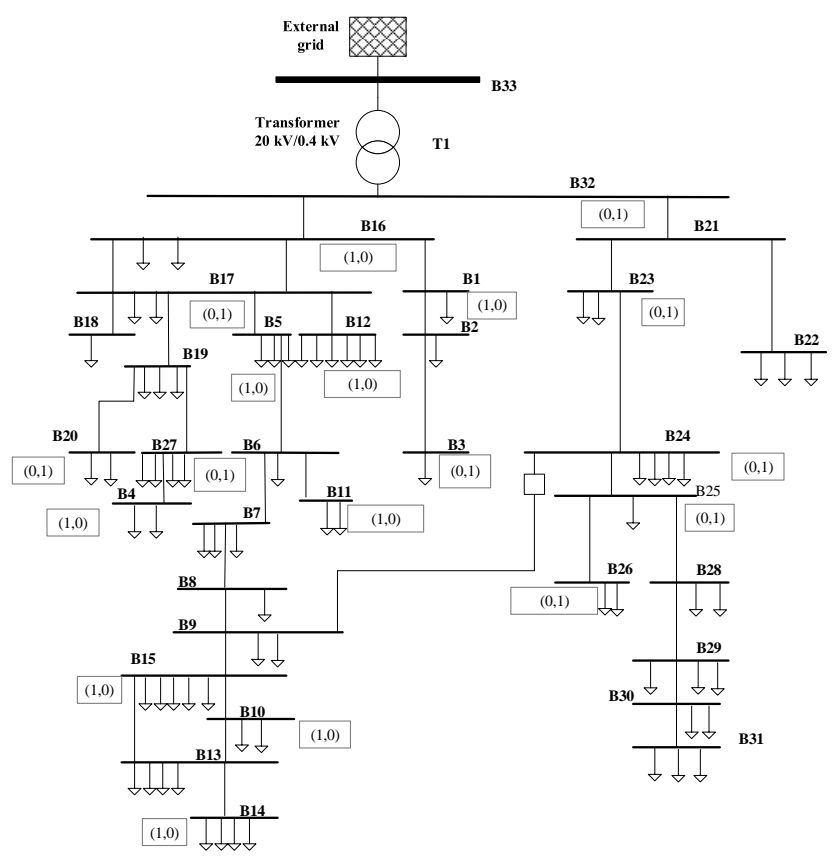

Fig. 6: A representative Danish distribution network. The parentheses indicate the number of connected EVs under each bus with the first number showing the number of Agg.1's EVs and the second Agg.2's EVs.

price difference of up-regulating power price and intra-day market prices, as illustrated in Fig. 7, the VSs tend to charge or discharge at few time slots. It is observed that both aggregators provide up-regulating power service to the system operator at the first hour of the optimization horizon. $180 \mathrm{~kW}$ of upregulating power can be provided by the two aggregators, which is a considerable power flow despite the relatively small number of aggregated EVs. Then, throughout the remainder of the day, this discharged power will be charged back in order to satisfy the SOC energy requirements at the end of the optimization horizon. The simulation shows that with proper management, EVs can be a significant service provider to the TSO. However, such large power deviations in a relatively short time interval may violate the operational constraints of the DNO, if the network is not dimensioned for such large power changes. In the following subsection, we show the effectiveness of transactive energy in preventing possible local network problems by the aggregators' participation in the balancing market.

\section{Aggregators' Modified Schedules after NCTE}

Fig. 7 shows the sum of two aggregators' power at the transformer level as well as the transformer capacity on both power flow directions. It is shown that before applying the NCTE, in a few time slots and on both power flow directions, the aggregated power of the two aggregators exceed the transformer capacity limits; these problems are resolved after applying the transactive energy method. To illustrate the transactive procedure, the power of two different time slots at bus 16 is selected. As presented in Fig. 8, the requested power 


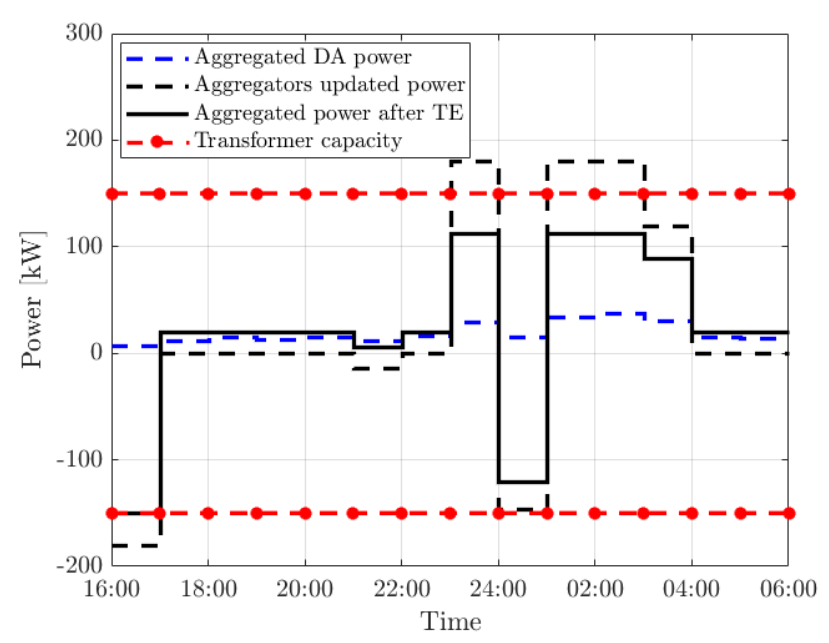

Fig. 7: The sum of the aggregators power before and after the transactive energy

of aggregators in the first time slot at a bus level is equal to $-10 \mathrm{~kW}$, which results (collectively with the requested power at the othe buses) in a violation of the transformer's power limit. The DNO's allowed capacity at the start of the iterations is approximately $-8.3 \mathrm{~kW}$. Similarly, at time slot 10 the aggregators wish to increase their consumption to 10 $\mathrm{kW}$, whereas the DNO allocates only $8.7 \mathrm{~kW}$. It is interesting to observe the impact of the quadratic cost functions in the convergence of the aggregators schedules and the allocated capacity by the DNO. The DISO generates the appropriate prices via the proposed NCTE framework to address these conflicts. Since the quadratic objective functions minimize the deviations from the unconstrained values for all time slots and buses, non-monotonic convergence of the aggregators' schedules is not uncommon (see time slot 1). After approximately 60 iterations the aggregators' schedules and the DNO's power values converge. We conducted extensive simulations and we found that in all cases the solution obtained by applying the NCTE method is the same as the one obtained by solving the centralized problem.

The impact of the EVs participation in the balancing market on the voltage levels is shown in Fig. 9. The voltage levels at bus 14 are shown for the whole optimization horizon and for 3 cases: (a) without the EVs participating in the balancing market, (b) with the EVs offering balancing power without applying NCTE and (c) after applying the NCTE. It is demonstrated that the NCTE method ensures that voltage stays within the allowed range while the EVs offer balancing power. Note that the voltages shown in Fig. 9 are calculated from the $\mathrm{AC}$ power flow calculations and at some time slots they are not exactly equal to the defined $0.9 \mathrm{pu}$ limit, due to the voltage approximation method used in this study. However, the very small differences indicate the effectiveness of the approximation.

\section{Receding Horizon Optimization Model}

To perform the receding horizon optimization, several parameters need to be updated which include: 1) equivalent SOC
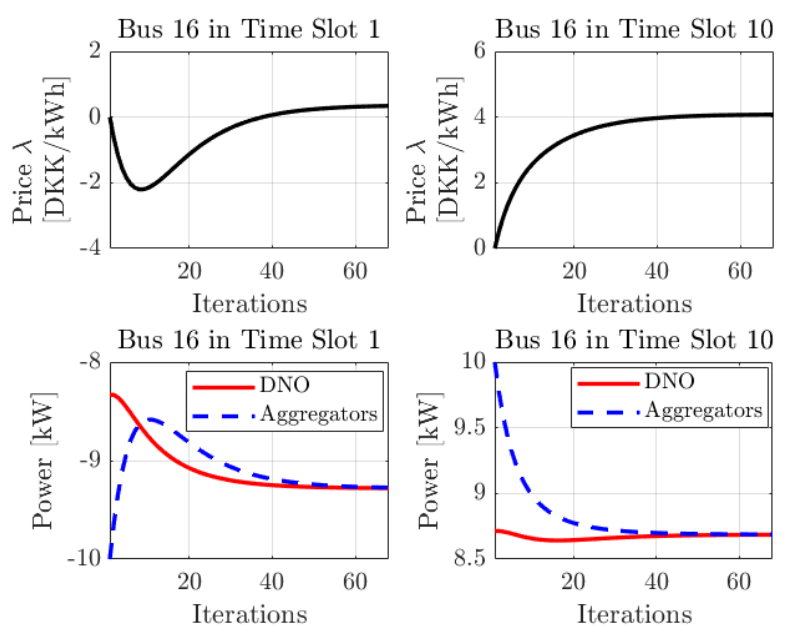

Fig. 8: Power and price convergence at Bus 16 in two different time slots.

states; 2) prediction of up/down regulating power price for the next time slot (in this case a down-regulating power price of $0.1 \mathrm{DKK} / \mathrm{kWh}$ is used); 3) the new initial charging/discharging plan of each virtual storage, i.e., the updated power schedules obtained in subsection IV-C will be used as the new initial base schedule. With these inputs, one can get the new unconstrained power schedules for the remaining horizon.

In Fig. 10, the blue curve shows the updated aggregated power profiles obtained by applying NCTE at the previous time slot (at hour 16.00) and corresponds to the black solid curve in Fig. 7. For the new horizon, it can be seen that both aggregators plan to provide down-regulating power service, which exceeds the transformer capacity and violates the voltage constraints (solid black curve). To solve this problem, NCTE is applied again, resulting in the profile indicated by the dashed black curve in the figure.

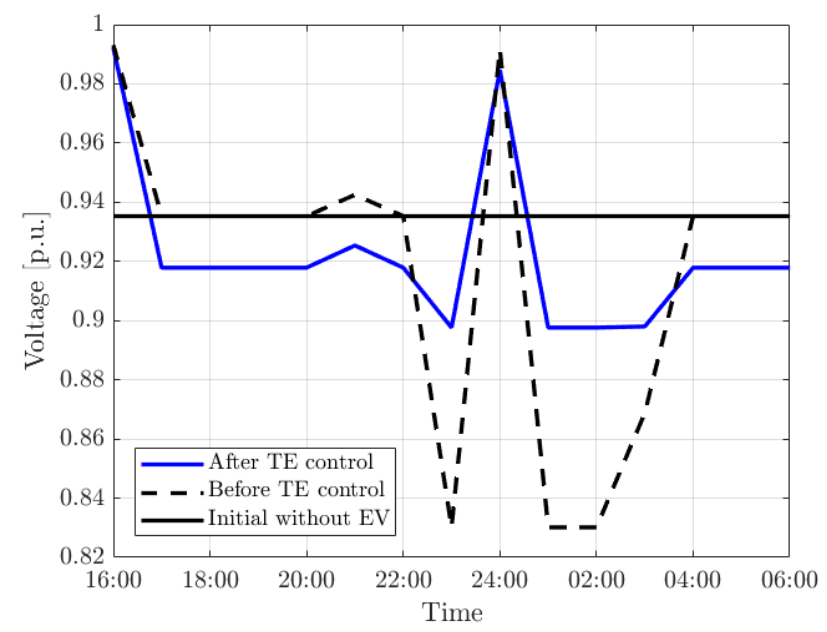

Fig. 9: Voltage at bus 14 for three cases: initial case without EV integration, before TE, after TE. 


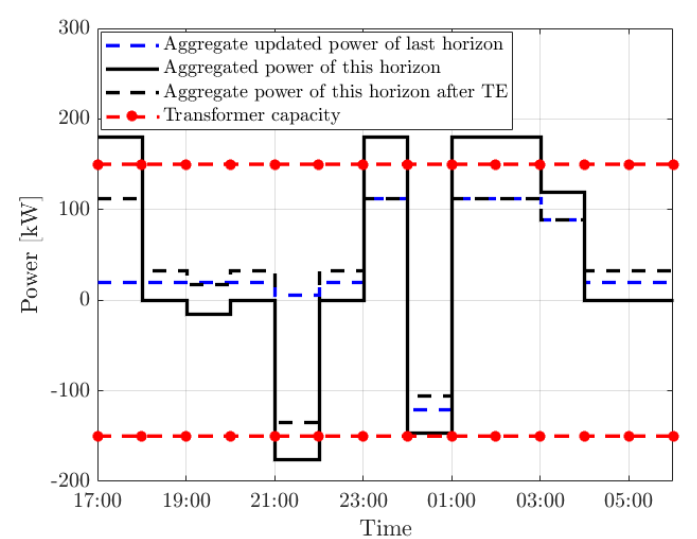

Fig. 10: The sum of the two aggregators DA and updated power profiles

\section{DiscuSSION AND CONCLUSION}

In this paper we proposed a market model that enables the aggregators' participation in the balancing market while avoiding congestion and voltage level violations in the distribution network. The model extends the NCTE model of our earlier work, with a connection to the balancing market framework of Nordic Europe. The paper provides the full optimization problem definition and elaborates on the implementation methods. The performance of the method was shown in a simulation case study using EV aggregators and a representative Danish distribution network. The study shows that the method actively solves distribution grid line congestion and voltage violation problems.

Using a transactive approach, potential conflicts between the TSO and the DNOs regarding prosumer flexibility usage can be resolved in a transactive manner, retaining user privacy. As both system operators compete for the available flexibility through a market-based framework, TSO/DNO conflicts are resolved on the basis of the valuation of the flexibility for the balancing market which is available at a certain time and grid location. For the DNO this entails an opportunity to solve network congestion in a new way, by using prosumer flexibility through the aggregators. For the aggregators, and their connected prosumers, this entails a new revenue stream for the available flexibility.

\section{REFERENCES}

[1] Y. Parag and B. K. Sovacool, "Electricity market design for the prosumer era," Nat. Energy, vol. 1, 2016.

[2] S. I. Vagropoulos and A. G. Bakirtzis, "Optimal bidding strategy for electric vehicle aggregators in electricity markets," IEEE Trans. Power Syst., vol. 28, no. 4, pp. 4031-4041, 2013.

[3] M. R. Sarker, Y. Dvorkin, and M. A. Ortega-Vazquez, "Optimal participation of an electric vehicle aggregator in day-ahead energy and reserve markets," IEEE Trans. Power Syst., vol. 31, no. 5, pp. 3506-3515, 2016.

[4] "Enel and italy's first vehicle-to-grid pilot." http://www.engerati.com/ article/italy-enel-nissan-first-vehicle-grid-V2G-charging. Accessed: 2017-08-17.

[5] B. Biegel, P. Andersen, T. S. Pedersen, K. M. Nielsen, J. Stoustrup, and L. H. Hansen, "Electricity market optimization of heat pump portfolio," in IEEE Int. Conf. Control App., pp. 294-301, IEEE, 2013.

[6] W. Pei et al., "Optimal bidding strategy and intramarket mechanism of microgrid aggregator in real-time balancing market," IEEE Trans. Ind. Informat., vol. 12, no. 2, pp. 587-596, 2016.
[7] N. Gatsis and G. B. Giannakis, "Residential load control: Distributed scheduling and convergence with lost ami messages," IEEE Trans. Smart Grid, vol. 3, no. 2, pp. 770-786, 2012.

[8] B. Moradzadeh and K. Tomsovic, "Two-stage residential energy management considering network operational constraints," IEEE Trans. Smart Grid, vol. 4, no. 4, pp. 2339-2346, 2013.

[9] M. K. Petersen et al., "A taxonomy for modeling flexibility and a computationally efficient algorithm for dispatch in smart grids," in Amer. Control Conf., pp. 1150-1156, IEEE, 2013.

[10] H. Hao, D. Wu, J. Lian, and T. Yang, "Optimal coordination of building loads and energy storage for power grid and end user services," IEEE Trans. Smart Grid, 2017.

[11] A. Pentland, "Simple market models fail the test," Nat., vol. 525, no. 7568, pp. 190-192, 2015.

[12] S. E. Widergren, J. M. Roop, R. T. Guttromson, and Z. Huang, "Simulating the dynamic coupling of market and physical system operations," in IEEE PES General Meeting, 2004., pp. 748-753 Vol.1, June 2004.

[13] J. K. Kok, C. J. Warmer, and I. G. Kamphuis, "PowerMatcher: Multiagent control in the electricity infrastructure," in Proc. 4th Int. Joint Conf. on Autonomous Agents and Multiagent Systems, AAMAS '05, (New York, NY, USA), pp. 75-82, ACM, 2005.

[14] K. Kok and S. Widergren, "A society of devices: Integrating intelligent distributed resources with transactive energy," IEEE Power Energy Mag., vol. 14, no. 3, pp. 34-45, 2016.

[15] J. Hu, G. Yang, K. Kok, Y. Xue, and H. W. Bindner, "Transactive control: a framework for operating power systems characterized by high penetration of distributed energy resources," J Mod Power Syst Cle, vol. 5, no. 3, pp. 451-464, 2016.

[16] J. Hu, G. Yang, H. W. Bindner, and Y. Xue, "Application of networkconstrained transactive control to electric vehicle charging for secure grid operation," IEEE Trans. Sustain. Energy, vol. 8, no. 2, 2017.

[17] C. A. Floudas, Nonlinear and mixed-integer optimization: fundamentals and applications. Oxford University Press, 1995.

[18] S. Gill, I. Kockar, and G. W. Ault, "Dynamic optimal power flow for active distribution networks," IEEE Transactions on Power Systems, vol. 29, pp. 121-131, Jan 2014.

[19] F. Marra, G. Yang, C. Trholt, J. stergaard, and E. Larsen, "A decentralized storage strategy for residential feeders with photovoltaics," IEEE Transactions on Smart Grid, vol. 5, pp. 974-981, March 2014.

[20] S. Bolognani and S. Zampieri, "On the existence and linear approximation of the power flow solution in power distribution networks," IEEE Transactions on Power Systems, vol. 31, pp. 163-172, Jan 2016.

[21] S. Boyd, L. Xiao, A. Mutapcic, and J. Mattingley, "Notes on decomposition methods," Notes for EE364B, Stanford University, Winter 2006-07, vol. 2007, 2008.

[22] S. Boyd and A. Mutapcic, "Subgradient methods," Lecture notes of EE364b, Stanford University, Winter Quarter, vol. 2007, 2006.

[23] S. Mhanna et al., "A fast distributed algorithm for large-scale demand response aggregation," IEEE Trans. Smart Grid, vol. 7, no. 4, 2016.

[24] "Gurobi optimizer." http://www.gurobi.com/.

[25] J. Löfberg, "Yalmip : A toolbox for modeling and optimization in matlab," in In Proceedings of the CACSD Conference, (Taipei, Taiwan), 2004.

[26] R. D. Zimmerman, C. E. Murillo-Sánchez, and R. J. Thomas, "Matpower: Steady-state operations, planning, and analysis tools for power systems research and education," IEEE Trans. Power Syst., vol. 26, no. 1, pp. 12-19, 2011.

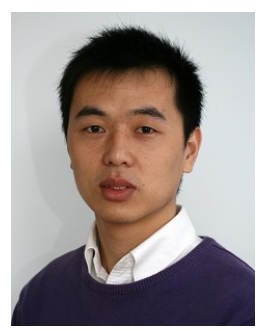

Junjie Hu (M14) received his M.Sc. degree in control theory and control engineering from Tongji University, China, in 2010, and the Ph.D. degree in electrical engineering from the Technical University of Denmark, Denmark, in 2014. He was a postdoc researcher with the Department of Electrical Engineering, Technical Unversity of Denmark. He is currently an Associate Professor with School of Electrical and Electronic Engineering, North China Electric Power University. His research interests include distributed energy resources energy management and their participations in frequency ancillary service markets. 


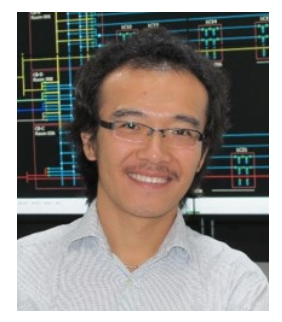

Guangya Yang (SM14) received B.E. and M.E. degree from Shandong University in 2002 and 2005 respectively, and $\mathrm{Ph} . \mathrm{D}$. degree in 2008 from the University of Queensland, all in the field of electric power system. He joined Technical University of Denmark from 2009 as Postdoctoral Researcher. Currently he is Associate Professor with the Center for Electric Power and Energy, Department of Electrical Engineering, Technical University of Denmark. Since 2009, he has been developing and leading industrial collaborative projects in Denmark in the field of monitoring, integration and protection of renewable energy based systems. His current research interests include operation of low inertia power systems, smart grids, and cyber-physical energy systems.

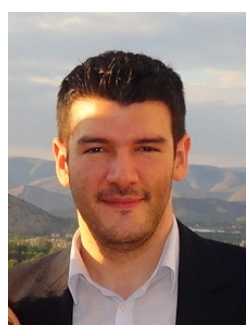

Charalampos Ziras (S16) received the Dipl.-Ing degree in electrical and computer engineering from the National Technical University of Athens, Athens, Greece, in 2009. He then worked as a Telecommunications Engineer on benchmarking and optimization of mobile networks. In 2015, he received the M.Sc. degree in energy science and technology from ETH Zurich (Swiss Federal Institute of Technology), Zurich, Switzerland. In 2016, he joined the Center for Electric Power and Energy, Technical University of Denmark (DTU), Lyngby, Denmark, where he is currently working towards his Ph.D. degree. His research interests include aggregation, optimization, and control of DERs for the concurrent provision of power system services, as well as DSO mechanisms for the integration of large shares of DERs in distribution networks.

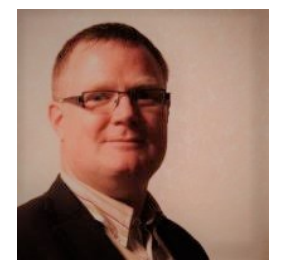

Koen Kok holds a BSc in Electrical Engineering, a BSc in Technical Informatics and an MSc in Computer Science, the latter from the University of Groningen in The Netherlands. In 2013, he received a Computer Science PhD from the VU University Amsterdam for his thesis on smart grid coordination mechanisms based on distributed software technology. Currently, Kok is Senior Scientist at $\mathrm{TNO}$, the largest applied research institute in The Netherlands, and he has a part-time position at het Technical University of Eindhoven. Formerly, Kok has worked for the Energy research Center of the Netherlands (ECN), the VU University Amsterdam and the Technical University of Denmark (DTU). Kok has extensive research experience in the fields of market-based control of power systems, smart grid ICT architectures and integration of distributed energy resources and demand response in the electricity system. Key results have been field deployed, commercialized and/or made available in opensource. 